\title{
Double-blind trial of the efficacy of pentoxifylline $v s$ thalidomide for the treatment of type II reaction in leprosy
}

A.M. Sales ${ }^{1}$, H.J. de Matos ${ }^{2}$, J.A.C. Nery ${ }^{1}$, N.C. Duppre ${ }^{1}$, E.P. Sampaio ${ }^{1}$ and E.N. Sarno ${ }^{1}$

\author{
${ }^{1}$ Laboratório de Hanseníase, Fundação Oswaldo Cruz, Rio de Janeiro, RJ, Brasil \\ 2Departamento de Tecnologia da Informação e Educação em Saúde, \\ Faculdade de Ciências Médicas, Universidade do Estado do Rio de Janeiro, \\ Rio de Janeiro, RJ, Brasil
}

Correspondence

E.N. Sarno

Laboratório de Hanseníase

FIOCRUZ

Av. Brasil, 4365

21045-900 Rio de Janeiro, RJ

Brasil

Fax: +55-21-2270-9997

E-mail: euzenir@fiocruz.br

Research supported by

UNDP/World Bank/TDR-WHO

$\ldots \ldots \ldots \ldots \ldots \ldots$

Received May 31, 2005

Accepted September 27, 2006

...................

\begin{abstract}
Type II reaction in leprosy, or erythema nodosum leprosum (ENL), is often characterized by severe clinical symptoms together with nerve function impairment leading to permanent disabilities. Thalidomide has been shown to be a highly effective drug for the treatment of ENL. It is, however, contraindicated for women of childbearing age due to its teratogenicity. On the other hand, pentoxifylline, used to treat hypercoagulable states, is not teratogenic and, like thalidomide, can inhibit the synthesis of tumor necrosis factor- $\alpha$ and other cytokines. In the present randomized double-blind clinical study we compared the effectiveness of orally administered pentoxifylline $v s$ thalidomide in treating type II reaction in 44 patients. Daily doses of $300 \mathrm{mg}$ thalidomide or $1.2 \mathrm{~g}$ pentoxifylline were administered for 30 days to multibacillary leprosy patients undergoing type II reaction. Randomly chosen patients were included in the study before, during, and after specific multidrug therapy. Clinical evaluations were performed on the 1st, 7th, 14th, 21st, and 30th days of treatment and laboratory tests were carried out on the 1st and 30th days. As expected, overall, thalidomide proved to be more effective in the treatment of type II leprosy reaction. Nevertheless, continuous treatment with pentoxifylline was effective in relieving the clinical signs of ENL, especially limb edema and systemic symptoms, in $62.5 \%$ of the patients.
\end{abstract}

Key words

- Leprosy

- Reactional episodes

- Therapy

- Pentoxifylline

- Thalidomide

\section{Introduction}

Reactional states are acute inflammatory events that commonly occur in leprosy during the course of the disease. Reactions are classified as type I, or reversal reaction, and type II, or erythema nodosum leprosum (ENL) (1). Rarely severe enough to require hospitalization, both types have been found to cause nerve inflammation (neuritis), representing the primary cause of irreversible deformities.

Known to occur in borderline-lepromatous and lepromatous-lepromatous patients, the frequency of ENL may vary. In a previous study, it was demonstrated that $57 \%$ of patients undergoing multibacillary multidrug therapy presented reaction, with 55\% having 
ENL (2). Type II reaction, characterized by the appearance of tender, erythematous, subcutaneous nodules located on apparently normal skin, is frequently accompanied by systemic symptoms such as fever, malaise, enlarged lymph nodes, anorexia, weight loss, arthralgia, and edema $(3,4)$. Additional organs, including the testes, joints, eyes, and nerves, may also be affected. Furthermore, a patient may present significant leukocytosis that typically recedes after the reactional state has subsided (5). Some reports have confirmed the presence of high levels of proinflammatory cytokines such as tumor necrosis factor- $\alpha$ (TNF- $\alpha$ ), IL- 6 , and IL- 1 in the sera of ENL patients, suggesting that these pleiotropic inflammatory cytokines may be at least partly responsible for the clinical manifestations of type II reaction $(6,7)$.

The treatment of ENL has been a controversial topic for many years (8). While clearly very effective, thalidomide has a teratogenic effect, causing it to be banned in many countries. However, although prohibited for women of childbearing age, the drug has been officially recommended by the Brazilian Leprosy Program of the Ministry of Health to treat type II leprosy reaction (9). In contrast, corticosteroids are the drugs of choice for ENL leprosy patients suffering from neuritis. The WHO has recommended the anti-inflammatory clofazimine (10) for chronic and severe ENL that does not respond satisfactorily to corticosteroid treatment or in cases in which the risk of toxicity is high. Many studies performed during the last 10 years have shown that thalidomide not only is a potent modulator of the immune response but also has a substantial beneficial effect in blocking the effects of TNF- $\alpha$, most probably a critical factor in the control of ENL $(11,12)$. It is known that the recognized side effects of thalidomide do not represent a serious threat and that peripheral neuropathy only rarely occurs.

Pentoxifylline, a methylxanthine derivative with potent hemorheologic-like proper- ties, was initially marketed to treat patients with intermittent claudication (13). Pentoxifylline, believed to have a potentially crucial effect on the control of ENL, blocks the synthesis of TNF- $\alpha$ messenger RNA by inhibiting gene transcription (14). In non-controlled studies, pentoxifylline has been found to be both well tolerated and effective in reducing local and systemic symptoms of ENL (15). Its key advantage is that it has been shown to have no teratogenic effects and, hence, can be taken by female patients of childbearing age without concern.

The present randomized, double-blind trial was carried out to determine the efficacy and safety profiles of pentoxifylline $v s$ thalidomide in treating ENL.

\section{Patients and Methods}

A randomized, double-blind trial was carried out on two groups of patients to compare the results of treatment regimens consisting of $300 \mathrm{mg}$ thalidomide vs $1.2 \mathrm{~g}$ pentoxifylline. The study was conducted at the Oswaldo Cruz Foundation (FIOCRUZ), Rio de Janeiro, RJ, Brazil, from February 1998 to June 2001 and was approved by the Ethics Committee of FIOCRUZ. All subjects gave informed written consent to participate in the study.

The following inclusion criteria were applied: 1) a diagnosis of multibacillary leprosy, 2) males between 18-60 years of age and females over 49 (postmenopausal), 3) clinical and histopathological data demonstrating the occurrence of a type II reaction, defined as inflammatory cutaneous nodules or polymorphic cutaneous lesions (erythema multiforme), 4) nerve examinations performed by an experienced neurologist that excluded any sign of acute neuritis requiring corticosteroid therapy, and 5) patients giving informed written consent.

Females under 49 and all leprosy patients suffering from hepatic, renal, or mental diseases, diabetes and/or immune-defi- 
ciencies related to HIV or not were excluded from the study.

Patients who showed no improvement after 15 days of treatment, or who presented any severe adverse effects related to ingestion of the medication, i.e., gastrointestinal disturbances (vomiting, diarrhea), headaches, and dizziness, or developed acute neuritis, were removed from the study. These patients were treated with regular regimen recommended by the Ministry of Health with thalidomide or corticosteroids. Furthermore, all patients were clinically evaluated on the $1 \mathrm{st}, 7 \mathrm{th}, 14 \mathrm{th}, 21 \mathrm{st}$, and 30th days of treatment. Blood was collected for tests that included a complete blood cell count and assessment of C-reactive protein (CRP) levels at the beginning and at the end of the study.

Clinical evaluation was based on the following parameters: axillary temperature and the presence of reactional cutaneous lesions, enlarged peripheral lymph nodes, edema, and other symptoms (nausea, vomiting, adynamia, headaches, arthralgia, myalgia). Patients were considered to be reaction free when, at the end of 30 days of treatment, the following criteria were satisfactorily met: complete elimination of type II reactional skin lesion inflammation, normal body temperature and/or regression of systemic symptoms.

Analyses were based on intention to treat. The resulting data were analyzed statistically using the Epi-Info 6.04 software program (Centers for Disease Control and Prevention, Atlanta, GA). The significance of the difference between proportions was tested by the Yates-corrected chi-square test. A P value of less than $5 \%$ was used as the level of statistical significance.

An outside observer assigned to periodically evaluate the results interrupted the study when a significant difference was found between the effects of the drugs.

After rigorous screening, 44 leprosy patients were selected for the study. Two were included twice since two independent reac- tional episodes were observed in these patients at separate 3-month intervals. Twenty group 1 patients received thalidomide and 24 group 2 patients were given pentoxifylline. In the thalidomide group, 19 of the 20 patients completed the full 30-day treatment while in the pentoxifylline group, only 17 of the 24 patients were able to complete treatment.

\section{Patients excluded during the treatment}

Of the 7 patients taking pentoxifylline, 1 had gastrointestinal intolerance to the drug in the form of nausea and intense vomiting; 2 had taken other medications (1 ingested pentoxifylline on his own and the other developed acute neuritis during the course of the protocol when corticosteroids were introduced); 3 presented fever and continuing lesion inflammation after 2 weeks of treatment; 1 pentoxifylline-treated patient did not return for weekly visits; 1 thalidomidetreated patient did not complete treatment. All of these patients were removed from follow-up and categorized as treatment nonresponders.

\section{Results}

The characteristics of the two groups are shown in Table 1. Table 2 provides the status of clinical and laboratory features on days 1 , $7,14,21$, and 30 . At the end of the study, $20 \%$ of thalidomide-treated and about $12 \%$ of pentoxifylline-treated patients continued to present enlarged lymph nodes. Pentoxifylline performed somewhat better than thalidomide in inducing regression of enlarged nodes and edema. In contrast, thalidomide was more successful in reducing inflammatory nodules typical of type II reaction. Among all patients analyzed during reaction, $91 \%$ presented cutaneous nodules. Notably, however, approximately $95 \%$ of thalidomide-treated and a much lower 59\% of pentoxifylline-treated patients showed skin 
Table 1. Characteristics of patients before treatment with thalidomide or pentoxifylline.

\begin{tabular}{lcc}
\hline & Thalidomide $(\mathrm{N}=20)$ & Pentoxifylline (N = 24) \\
\hline $\begin{array}{l}\text { Age 18-69 (average) } \\
\text { Gender }\end{array}$ & 37.1 years & 33.4 years \\
$\quad$ Male & & \\
Female & $85 \%(17)$ & $87.5 \%(21)$ \\
Disability grade & $15 \%(3)$ & $12.5 \%(3)$ \\
0 & & \\
1 & $40 \%(8)$ & $45.8 \%(11)$ \\
2 & $35 \%(7)$ & $29.2 \%(7)$ \\
Clinical form & $25 \%(5)$ & $25 \%(6)$ \\
BL & & $16.7 \%(4)$ \\
LL & $30 \%(6)$ & $83.3 \%(20)$ \\
Bacillary index (average) & $70 \%(14)$ & 2.42
\end{tabular}

Data are reported as percent with number of patients in parentheses. $\mathrm{BL}=$ borderlinelepromatous; $\mathrm{LL}=$ lepromatous-lepromatous. There were no statistical differences between groups (chi-square test, $\alpha=0.05$ ).

Table 2. Clinical and laboratory features of type II reaction upon initial examination and their evolution during treatment.

\begin{tabular}{|c|c|c|c|c|c|}
\hline Clinical and laboratory features & Day 1 & Day 7 & Day 14 & Day 21 & Day 30 \\
\hline \multicolumn{6}{|l|}{ Inflammatory skin nodules } \\
\hline Thalidomide & $70 \%(14)$ & $5 \%(1)$ & $5 \%(1)$ & $5 \%(1)$ & $10 \%(2)$ \\
\hline Pentoxifylline & $65 \%(11)$ & $41 \%(7)$ & $35 \%(6)$ & $23 \%(4)$ & $12 \%(2)$ \\
\hline \multicolumn{6}{|l|}{ Temperature $\geq 37.6$} \\
\hline Thalidomide & $14 \%(3)$ & $5 \%(1)$ & $11 \%(2)$ & $0 \%$ & $0 \%$ \\
\hline Pentoxifylline & $9 \%(2)$ & $0 \%$ & $11 \%(2)$ & $6 \%(1)$ & $7 \%(1)$ \\
\hline \multicolumn{6}{|l|}{ Edema } \\
\hline Thalidomide & $55 \%(11)$ & $50 \%(10)$ & $50 \%(10)$ & $45 \%(9)$ & $40 \%(8)$ \\
\hline Pentoxifylline & $40 \%(7)$ & $30 \%(5)$ & $18 \%(3)$ & $30 \%(5)$ & $18 \%(3)$ \\
\hline \multicolumn{6}{|l|}{ Enlarged lymph nodes } \\
\hline Thalidomide & $30 \%(6)$ & $20 \%(4)$ & $15 \%(3)$ & $20 \%(4)$ & $20 \%(4)$ \\
\hline Pentoxifylline & $41 \%(7)$ & $23 \%(4)$ & $6 \%(1)$ & $6 \%(1)$ & $12 \%(2)$ \\
\hline \multicolumn{6}{|l|}{ Other symptoms } \\
\hline Thalidomide & $75 \%(15)$ & $35 \%(7)$ & $30 \%(6)$ & $35 \%(7)$ & $25 \%(5)$ \\
\hline Pentoxifylline & $59 \%(10)$ & $53 \%(9)$ & $35 \%(6)$ & $44 \%(7)$ & $29 \%(5)$ \\
\hline \multicolumn{6}{|l|}{ White blood cells $\geq 11,000$} \\
\hline Thalidomide & $29 \%(6)$ & - & - & - & $0 \%$ \\
\hline Pentoxifylline & $45 \%(10)$ & - & - & - & $8 \%(1)$ \\
\hline \multicolumn{6}{|l|}{ Positive C-reactive protein } \\
\hline Thalidomide & $72 \%(13)$ & - & - & - & $33 \%(6)$ \\
\hline Pentoxifylline & $60 \%(9)$ & - & - & - & $20 \%(3)$ \\
\hline
\end{tabular}

Data are reported as percent with number of patients in parentheses. Laboratory tests were carried out only at the beginning and the end of the study. lesion regression after 7 days. The analysis of other symptoms occurring during the reaction revealed that $74 \%$ of all patients suffered from nausea, vomiting, headaches, adynamia, myalgia, and/or arthralgia. It was also found that among the $75 \%$ of thalidomide-treated patients presenting other symptoms at diagnosis (day 1), only $25 \%$ presented these same symptoms on day 30 . Likewise, in pentoxifylline-treated patients, among the $59 \%$ presenting other symptoms at diagnosis, only $29 \%$ maintained the same symptomatology at the end of the study.

The results of white blood cell count and the positivity of CRP levels were found to vary more frequently. In about $45 \%$ of thalidomide-treated and $29 \%$ of pentoxifyllinetreated patients, leukocytosis (more than 11,000 white blood cells $/ \mathrm{mm}^{3}$ ) was observed at the initial examination even though regression occurred in both groups. CRP levels were found to be positive in $72 \%$ of group 1 and $60 \%$ of group 2 patients, whereas on day 30 the percentage of thalidomidetreated and pentoxifylline-treated patients presenting a positive test had sharply declined to 33 and $20 \%$, respectively.

To evaluate the performance of each drug and compare the respective data, analyses were carried out to measure their effectiveness after 2 weeks and at the end of treatment. Table 3 summarizes and grades the results as follows: 1) partial improvement when 1 or more symptoms were manifested; 2) total improvement when $100 \%$ improvement occurred after a type II reactional episode; 3) general improvement considering both groups together (partial improvement + total improvement). As shown by an intention to treat analysis, at the end of 2 weeks patients taking thalidomide showed a general improvement rate of $95 \%$ while those taking pentoxifylline obtained a $75 \%$ rate. However, at the end of 4 weeks, whereas the general improvement rate of the thalidomide group remained at $95 \%$, that of the pentoxifylline group dropped to $62.5 \%$. When these 
data were compared by the Yates-corrected chi-square method at 4 weeks, there was a statistically significant difference between the drugs $(\mathrm{P}=0.02)$.

Most of the adverse reactions caused by each drug, especially those related to gastrointestinal complaints and nausea, occurred during the first week, tending to completely disappear over the remaining weeks of treatment. Only 1 pentoxifylline patient discontinued treatment due to the severity of these adverse effects. No significant difference in the occurrence of adverse reactions was detected between the two drugs (at the end of the first week and at the end of the third week; see Table 4).

\section{Discussion}

It is clear that the results of this doubleblind controlled study strongly support the beneficial effects of pentoxifylline in treating $\operatorname{ENL}(15,16)$. However, at the end of 1 month of treatment, the clinical results showed that the overall response to thalidomide was significantly superior to the response to pentoxifylline. At the end of treatment, the improvement rate of patients taking pentoxifylline was a noteworthy $62.5 \%$ compared to $95 \%$ for those taking thalidomide. Furthermore, thalidomide acted more rapidly in reducing skin lesions. By day 7 , only $5 \%$ of the patients presented skin lesions $v s 41 \%$ of those taking pentoxifylline. In agreement with these results, in a clinical trial with thalidomide and acetylsalicylic acid, Iyer et al. (17) demonstrated that, by day 8 , skin lesions had disappeared in $73 \%$ of the ENL reactional patients treated with thalidomide. In our study, pentoxifylline also contributed remarkably to the regression of edema and lymph node enlargement.

It is known that ENL patients often present severe leukocytosis capable of attaining a maximum of $30,000 / \mathrm{mm}^{3}$, characteristic of a leukemoid reaction (6). Oliveira et al. (18) have shown that, in ENL patients, poly- morphonuclear neutrophils participated in the acute inflammatory response as an additional source of TNF- $\alpha$, correlating to the intense neutrophil infiltrate in these lesions. In the present study, the leukocyte count similarly regressed as a consequence of treatment with both drugs.

Foss et al. (19) have reported high CRP levels along with a positive correlation (95\%) between elevated TNF- $\alpha$ and CRP levels in the serum of ENL patients. In the present study, on day 1 , a high percentage of the ENL patients in both groups presented high serum levels of CRP (72\% of group 1 and $60 \%$ of group 2) that regressed by day 30 . Taken together, leukocytosis and CRP could be considered as laboratory parameters to be used to follow up the systemic inflammatory response and the decrease in serum TNF- $\alpha$ levels $(18,19)$.

Table 3. Comparison of the effectiveness of treatment on the clinical signs and symptoms of leprosy type II reaction between thalidomide-treated and pentoxifyllinetreated patients.

\begin{tabular}{|c|c|c|c|c|c|c|}
\hline & \multicolumn{3}{|c|}{15 days } & \multicolumn{3}{|c|}{30 days } \\
\hline & $\mathrm{PI}$ & $\mathrm{TI}$ & $\mathrm{GI}(\mathrm{Pl}+\mathrm{TI})$ & $\mathrm{PI}$ & TI & $\mathrm{GI}(\mathrm{PI}+\mathrm{TI})$ \\
\hline Thalidomide & $60 \%$ & $35 \%$ & $95 \%$ & $50 \%$ & $45 \%$ & $95 \% *$ \\
\hline Pentoxifylline & $58.3 \%$ & $16.7 \%$ & $75 \%$ & $29.2 \%$ & $33.3 \%$ & $62 \%$ \\
\hline
\end{tabular}

In this intention to treat analysis, the randomized schedule was followed rigorously. $\mathrm{PI}=$ partial improvement; $\mathrm{TI}=$ total improvement; $\mathrm{GI}$ = general improvement . ${ }^{*} \mathrm{P}=0.02$, comparison of the two groups at 30 days ( $t$-test).

Table 4. Adverse reactions to thalidomide and pentoxifylline at the end of weeks 1 and 3.

\begin{tabular}{lccrrr}
\hline \multirow{2}{*}{ Adverse reactions } & \multicolumn{2}{c}{ End of 1st week } & & \multicolumn{2}{c}{ End of 3rd week } \\
\cline { 2 - 3 } \cline { 6 - 7 } & $\begin{array}{c}\text { Thalidomide } \\
(\mathrm{N}=20)\end{array}$ & $\begin{array}{c}\text { Pentoxifylline } \\
(\mathrm{N}=23)\end{array}$ & & $\begin{array}{c}\text { Thalidomide } \\
(\mathrm{N}=19)\end{array}$ & $\begin{array}{r}\text { Pentoxifylline } \\
(\mathrm{N}=18)\end{array}$ \\
\hline Presence & $5(25 \%)$ & $7(30.4 \%)$ & & $3(15.8 \%)$ & $2(11.1 \%)$ \\
Absence & $15(75 \%)$ & $16(69.6 \%)$ & & $16(64.2 \%)$ & $16(88.9 \%)$ \\
\hline
\end{tabular}

Data are reported as number of patients with percent in parentheses. There were no statistical differences between drugs at the end of the 1st or 3rd week (chi-square test). 
The present results obtained in the clinical evaluations carried out at the end of 30 days of treatment clearly indicated that the overall response to thalidomide was significantly better than the response to pentoxifylline. However, pentoxifylline could represent a good alternative treatment for ENL patients for whom thalidomide is contraindicated.

\section{Acknowledgments}

We would especially like to thank Rita Maria de Oliveira Pereira and Denise Vieira de Moura for technical assistance throughout the study, and Judy Grevan for editing the text. But, most of all, we would like to extend our gratitude to the patients themselves, who unfailingly gave their full and abiding cooperation whenever solicited.

\section{References}

1. Jolliffe DS. Leprosy reactional states and their treatment. $\mathrm{Br} J$ Dermatol 1977; 97: 345-352.

2. Nery JA, Vieira LM, de Matos HJ, Gallo ME, Sarno EN. Reactional states in multibacillary Hansen disease patients during multidrug therapy. Rev Inst Med Trop São Paulo 1998; 40: 363-370.

3. Sehgal VN. Reactions in leprosy. Clinical aspects. Int $J$ Dermatol 1987; 26: 278-285.

4. Stanley JN, Pearson JM, Ridley DB. Episodic edema in type 2 lepra reaction can be caused by transient lymphatic obstruction in the lymph node. Int $J$ Lepr Other Mycobact Dis 1986; 54: 231-235.

5. Rea TH, Levan NE. Erythema nodosum leprosum in a general hospital. Arch Dermatol 1975; 111: 1575-1580.

6. Parida SK, Grau GE, Zaheer SA, Mukherjee R. Serum tumor necrosis factor and interleukin 1 in leprosy and during lepra reactions. Clin Immunol Immunopathol 1992; 63: 23-27.

7. Sampaio EP, Sarno EN, Galilly R, Cohn ZA, Kaplan G. Thalidomide selectively inhibits tumor necrosis factor alpha production by stimulated human monocytes. J Exp Med 1991; 173: 699-703.

8. Pannikar V. The return of thalidomide: new uses and renewed concerns. Lepr Rev 2003; 74: 286-288.

9. Fundação Nacional da Saúde. Guia de controle da hanseníase. 2nd edn. Brasília: Ministério da Saúde; 1994.

10. WHO. WHO Expert Committee on Leprosy. Technical report series 874. Geneva: WHO 7th report; 1998.

11. Moreira AL, Sampaio EP, Zmuidzinas A, Frindt P, Smith KA, Kaplan $G$. Thalidomide exerts its inhibitory action on tumor necrosis factor alpha by enhancing mRNA degradation. J Exp Med 1993; 177: 1675-1680.

12. Sampaio EP, Kaplan G, Miranda A, Nery JA, Miguel CP, Viana SM, et al. The influence of thalidomide on the clinical and immunologic manifestation of erythema nodosum leprosum. J Infect Dis 1993; 168: 408-414.

13. Samlaska CP, Winfield EA. Pentoxifylline. J Am Acad Dermatol 1994; 30: 603-621.

14. Sarno EN, Nery JA, Garcia CC, Sampaio EP. Is pentoxifylline a viable alternative in the treatment of ENL? Int $J$ Lepr Other Mycobact Dis 1995; 63: 570-571.

15. De Carsalade GY, Achirafi A, Flageul B. Pentoxifylline in the treatment of erythema nodosum leprosum: results of an open study. Acta Leprol 2003; 12: 117-122.

16. Guinto RS, Tolentino JG, Malabay MC. Observations on erythema nodosum leprosum: Clinical evaluation studies in lepromatous leprosy. Int J Lepr Other Mycobact Dis 1963; 31: 81-94.

17. Iyer CG, Languillon J, Ramanujam K, Tarabini-Castellani G, De las Aguas JT, Bechelli LM, et al. WHO co-ordinated short-term doubleblind trial with thalidomide in the treatment of acute lepra reactions in male lepromatous patients. Bull World Health Organ 1971; 45: 719732.

18. Oliveira RB, Moraes MO, Oliveira EB, Sarno EN, Nery JA, Sampaio EP. Neutrophils isolated from leprosy patients release TNF-alpha and exhibit accelerated apoptosis in vitro. J Leukoc Biol 1999; 65: 364-371.

19. Foss NT, de Oliveira EB, Silva CL. Correlation between TNF production, increase of plasma C-reactive protein level and suppression of $\mathrm{T}$ lymphocyte response to concanavalin A during erythema nodosum leprosum. Int J Lepr Other Mycobact Dis 1993; 61: 218226. 